\title{
Investigation of Horizontal Velocity Fields in Stirred Vessels with Helical Coils by PIV
}

\author{
Volker Bliem ${ }^{1}$ and Heyko Jürgen Schultz ${ }^{2}$ \\ ${ }^{1}$ Lehrstuhl für Technische Chemie II, Universität Duisburg-Essen, 45117 Essen, Germany \\ ${ }^{2}$ Institute for Coatings and Surface Chemistry (ILOC), University of Applied Sciences Niederrhein, \\ Chemical Engineering/Faculty of Chemistry, Alderstraße 32, 47798 Krefeld, Germany \\ Correspondence should be addressed to Heyko Jürgen Schultz; heyko_juergen.schultz@hs-niederrhein.de
}

Received 23 January 2014; Accepted 27 April 2014; Published 19 May 2014

Academic Editor: Miguel J. Bagajewicz

\begin{abstract}
Copyright (C) 2014 V. Bliem and H. J. Schultz. This is an open access article distributed under the Creative Commons Attribution License, which permits unrestricted use, distribution, and reproduction in any medium, provided the original work is properly cited.

Horizontal velocity flow fields were measured by particle image velocimetry for a stirred vessel with baffles and two helical coils for enlargement of heat transfer area. The investigation was carried out in a cylindrical vessel with flat base and two different stirrers (radial-flow Rushton turbine and axial-flow propeller stirrer). Combined velocity plots for flow fields at different locations are presented. It was found that helical coils change the flow pattern significantly. Measurements for the axial-flow Rushton turbine showed a strong deflection by the coils, leading to a mainly tangential flow pattern. Behind baffles large areas of unused heat transfer area were found. First results for the axial-flow propeller reveal an extensive absence of fluid movement in the horizontal plane. Improved design considerations for enhanced heat transfer by more compatible equipment compilation are proposed.
\end{abstract}

\section{Introduction}

Mixing and stirring processes constitute widely spread and very important unit operations in chemical and allied industries. Most chemical products are treated at least once by a stirring process in an agitated vessel during their lifetime. The rotating impeller within a cylindrical vessel generates a very complex, three-dimensional flow structure, which is difficult to measure and quantify. Therefore, the mixing processes are not understood well in spite of many decades of utilization and scaling being done by means of semiempirical methods. Thus, solving and mixing-related problems in chemical plants are usually underrated. Based on estimates of Tatterson et al. [1] amounts of \$1-20 billion/year are lost due to mixingrelated problems only in the USA.

Special cases of chemical engineering are stirred vessels equipped with inserts of helical coils. These are helically, coiled pipes built into the vessel with the goal of achieving a higher heat exchange area. A heat transfer medium is conveyed through the additional coils, thereby increasing the indirect heat transfer between the medium in the vessel and outer heat transfer medium. This approach can, for example, have safety reasons or is used as a means to reduce reaction/process times. Especially in endo- and exothermic processes, such as distillations or polymerization reactions, the heat transfer area provided by the vessel jacket is not sufficient to guarantee a safe and economic process. Coils, therefore, disturb the flow structure within the vessel significantly. First experiments on coils in agitated vessels have been conducted by Chilton et al. [2] with a flat-paddle impeller. Nagata and Takhimoto [3], Appleton and Brennan [4], and Oldshue [5] expanded their work in this field on the wides pread Rushton turbine impeller. Oldshue [5], therefore, varied the dimensions of impeller and coil diameter and gave first recommendations on coil design. Skelland and Dimmick [6] examined the flow structure of a propeller impeller on Newton- and non-Newton fluids, however, with a deviating geometry compared to other authors. Most studies deal with and recommend radial-flow impellers for application with helical coils. Poggemann et al. [7] and Kraume [8] give a comprehensive overview. A direct comparison of radial and axial-flow impellers was conducted by Perarasu et al. [9], showing a considerably smaller heat transfer using the axialflow propeller impeller. 
This study investigates the flow structures of axial and radial-flow impellers in vessels with helical coils using twodimensional particle image velocimetry (2D-PIV). As a nonintrusive measuring technology to investigate complex flow fields, the application of PIV in science and industry gained significant importance during the past two decades [10]. PIV-studies on the flow field for a pitched blade turbine have been conducted by Sheng et al. [11], Myers et al. [12], and Bakker et al. [13], for the Rushton turbine by Hill et al. [14], Sharp and Adrian [15], Deen and Hjertager [16], Hill et al. [17], and Wang et al. [18], with increasing focus on the small scale flow around the impeller. Therefore, Atibeni et al. [19] studied the adverse effect of inserts in stirred vessels by use of 2D-PIV and showed the applicability of the technique for investigating flow fields in stirred vessels.

Besides experimental investigations, the numerical simulation of stirred tanks has become a powerful tool of chemical engineering. By the application of computational fluid dynamics (CFD) researchers can not only save much money through simulation-based, targeted vessel design and minimizing lab-plant studies but also get data which cannot be obtained from experiments. First simulations of the 2D flow field in stirred tanks were conducted by Harvey and Greaves [20]. Markatos [21] simulated the mixing of two liquids under the influence of different stirrer geometries and presented theoretical predictions of velocities and turbulence energy. Since then, the number of articles on the flow field in stirred tanks via CFD has increased significantly and established its own field of research. Although modern computer technology allows the full three-dimensional simulation of a stirred tank involving heat balances and chemical reactions, the validation of simulation results against experimental measurements is still evident. Here, PIV provides necessary guidance in turbulence modeling and defining boundary conditions as well as validation and improvement of CFD simulations. These two areas, CFD and PIV experiments, complement each other perfectly and can lead to a validated model for complex stirred tank reactor design in a medium term.

\section{Methodology}

2.1. Tank and Coil Geometry. The present mixing vessel model with helical coils was built with respect to typical recommendations on tank reactor design in industrial processes. The present experiments were conducted in a flat-bottomed, cylindrical mixing tank made of borosilicate glass with an inner diameter $\left(d_{1}\right)$ of $210 \mathrm{~mm}$. Two helically coiled pipes of a cupronickel alloy were placed into the tank (Figure 1). These were mounted as inner and outer coils on two square aluminum baffles, reaching from the bottom to the top of the tank. The vertical gap between the pipes of one coil was two times the pipe diameter $\left(d_{\text {Pipe }}\right)$, with an alternating arrangement of the two coils (Figure 2). Specific geometric dimensions of all parts are given in Table 1 . The tank was then filled with deionized water until the upper end of the coils. The filling height was equal to the tank diameter $\left(h_{0}=d_{1}\right)$.

The cylindrical tank was then placed in a larger, square glass tank. The space between the two tanks was filled
TABLE 1: Geometric dimensions of tank and coils.

\begin{tabular}{lccl}
\hline Symbol & Value & Unit & Comment \\
\hline$d_{1}$ & 210 & $\mathrm{~mm}$ & Inner tank diameter \\
$d_{\mathrm{i} C}$ & 144 & $\mathrm{~mm}$ & Inner diameter of inner coil \\
$d_{\mathrm{oC}}$ & 177 & $\mathrm{~mm}$ & Inner diameter of outer coil \\
$d_{P}$ & 4.7 & $\mathrm{~mm}$ & Pipe diameter \\
$n_{C}$ & 17 & & Number of turns \\
$m_{\mathrm{C}}$ & 9.5 & $\mathrm{~mm} / \mathrm{Turn}$ & Coil pitch \\
$h_{\mathrm{C}}$ & 43 & $\mathrm{~mm}$ & Bottom clearance of lowest coil \\
$h_{1}$ & 50 & $\mathrm{~mm}$ & Bottom clearance of stirrer \\
\hline
\end{tabular}

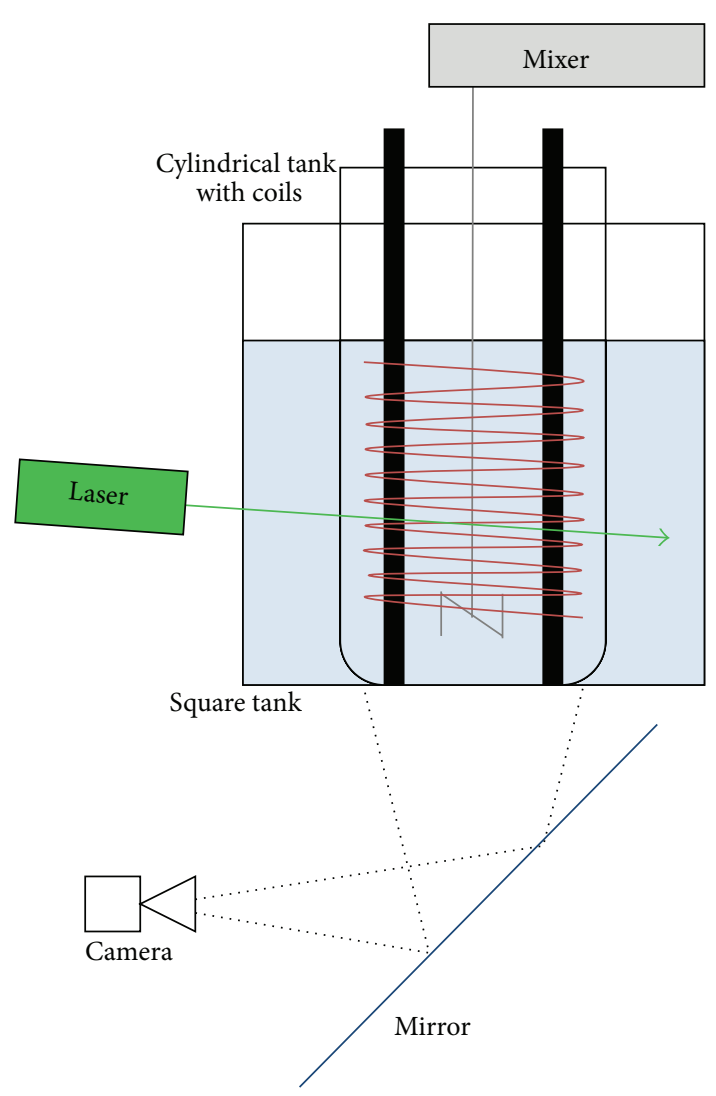

Figure 1: Side view schematic of experimental setup.

with deionized water to reduce optical distortions due to reflections on the curved surface of the cylindrical tank.

2.2. Experimental Setup. In this work, velocity plots of the $x$-and $y$-directions of three horizontal planes are presented. Due to the surrounding coils the view into the inside of the tank was hindered significantly. Therefore the PIV technique demands two separate optical accesses to work: one is for the laser-beam to illuminate the particles and one is for the camera perpendicular to the generated light sheet to map the illuminated particles (Figure 3). The first access was given by a slightly inclined light sheet through the gap between two pipes. The light sheets were applied successively at three heights: a low shot (LS), middle shot (MS), and top shot (TS) at 35,95 , and $172 \mathrm{~mm}$ bottom clearance, respectively. The 


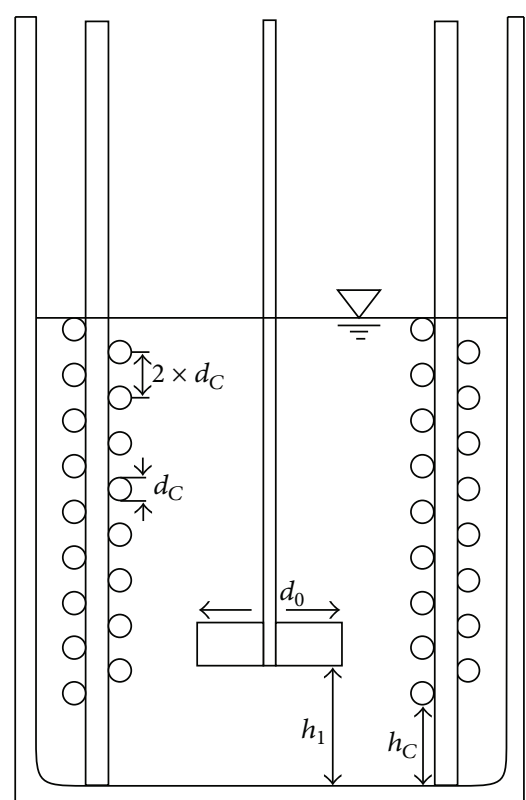

FIGURE 2: Geometric parameters for a stirred vessel with coils.

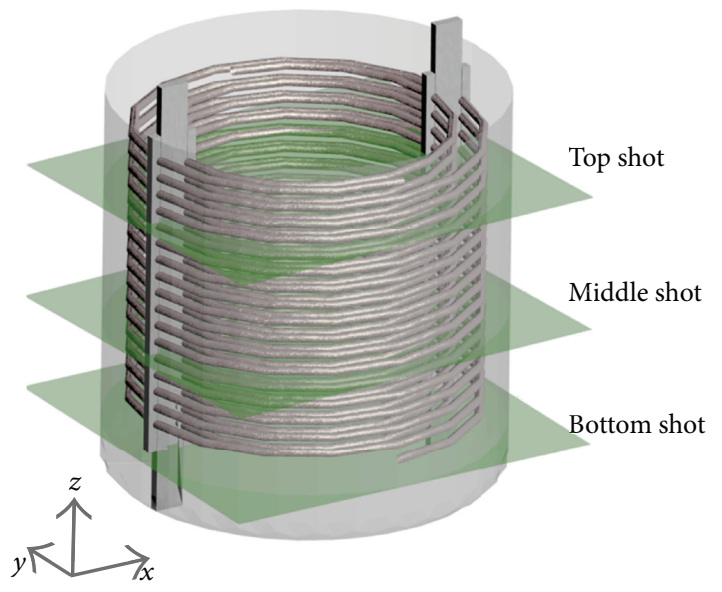

FIGURE 3: Computer model of the investigated stirred vessel with coils. The green planes indicate the location of the light sheets generated by the PIV-Laser.

inclination for middle and bottom shot was $-1.40^{\circ}$ around the $x$-axis and $2.54^{\circ}$ around the $y$-axis in accordance with the coil pitch. For the low shot no inclination of the laser was necessary since this shot was below the coils. For generation of the light beam a Nd:Yag laser emitting light with a wavelength of $532 \mathrm{~nm}$ provided by Dantec Dynamics, Ulm, was used.

The second access was accomplished through the transparent bottoms of mixing and glass tank by deflecting the camera view with a mirror. Images were taken by a FlowSenseEO $4 \mathrm{M}$ camera of Dantec Dynamics, containing 4 million pixels $(2,048 \times 2,048)$. The images were captured at 8 bit with respect to Adrian [10], clearly describing that an increased bit resolution does not lead to an increased accuracy, with a resulting pixel size of $107 \mu \mathrm{m}$. The camera frames and laser pulses were triggered by a 80 N77 Timer Box. The time between two laser pulses $(d t)$ was varied with the velocity within the mixing tank. The flow was seeded with polyamide particles of $d_{50}=54 \mu \mathrm{m}$.

2.3. Stirrers and Procedure. Mixing of the tank was provided by two different agitators: a down-pumping propeller agitator $\left(d_{0}=53 \mathrm{~mm}\right)$ and a Rushton turbine agitator $\left(d_{0}=52 \mathrm{~mm}\right)$ mounted central with a bottom clearance of $50 \mathrm{~mm}$ as can be seen in Figure 4 . Both agitators were driven by a RZR 2051 lab stirrer from Heidolph, Schwabach, in the range of 300 to $600 \mathrm{rpm}$. For each experimental run 200 images were captured to calculate the mean velocity by adaptive correlation algorithm of DynamicStudio Version 3.31. The recorded images were divided into initial interrogation areas (IA) of $128 \times 128$ pixels with two refinement steps up to $32 \times 32$ pixels and $50 \%$ overlap, resulting in $127 \times 127$ vectors for the entire cross-section.

\section{Results and Discussion}

3.1. Horizontal Cross-Section Velocity Field. In the following plots, contour surfaces of the velocity magnitude $|\vec{w}|$ combined with vector plots are used to visualize regions and directions of major flows in the horizontal plane within the tank. The velocity magnitude is coded by a color bar next to the plot and given by

$$
|\vec{w}|=\sqrt{w_{x}^{2}+w_{y}^{2}}
$$

where $w_{x}$ and $w_{y}$ are the velocity components in $x$ - and $y$ directions, respectively. To reduce the computing time and prevent misinterpretation, areas of no particle movement were masked and removed before calculating the velocity vectors. This includes areas between two pipes of one coil shadowed by the coils for the bottom view of the camera, areas behind the baffles, and areas outside the vessel. Areas which are either masked or where no valid velocity vectors were found remained white. Figure 5 shows the obtained full crosssection of the horizontal velocity field at $h / h_{0}=0.45$ or $95 \mathrm{~mm}$ bottom clearance (middle shot) for the Rushton turbine. The cross-section can be divided into three distinct areas: an inner circle and the two surrounding rings, each delimited by the inner and outer coils, recognizable as small white lines. The inner circle represents the velocity vectors in the horizontal plane at the given height of the inner cylinder. Besides, one can find velocity vectors in the inner and outer rings of the annulus between coil-coil and coil-vessel walls, respectively.

At $y=110$ the imaginary line along the two baffles builds a symmetry plane, dividing the measured cross-section flow field in the rear and front parts in the direction of the entering light sheet. Due to the slight skew position of the light sheet within the reactor, the received velocity fields are not perfectly symmetrical. The difference in height for the entering and leaving light sheet is $18 \mathrm{~mm}$ and can be assumed to be negligible towards the filling height of $210 \mathrm{~mm}$. Nevertheless, front and rear parts deviate only slightly; most 


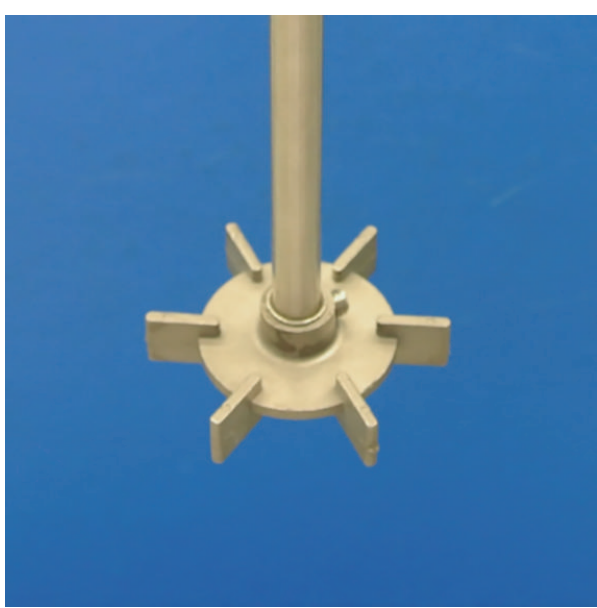

(a)

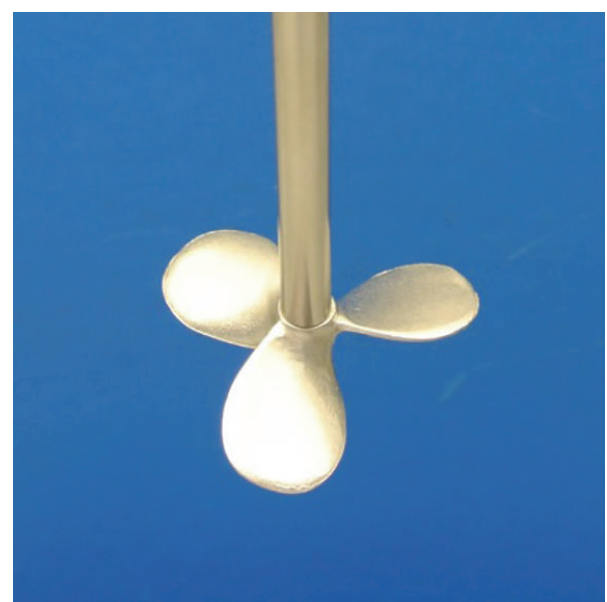

(b)

FIgURE 4: Pictures of the used agitators. (a) Rushton turbine and (b) propeller stirrer.

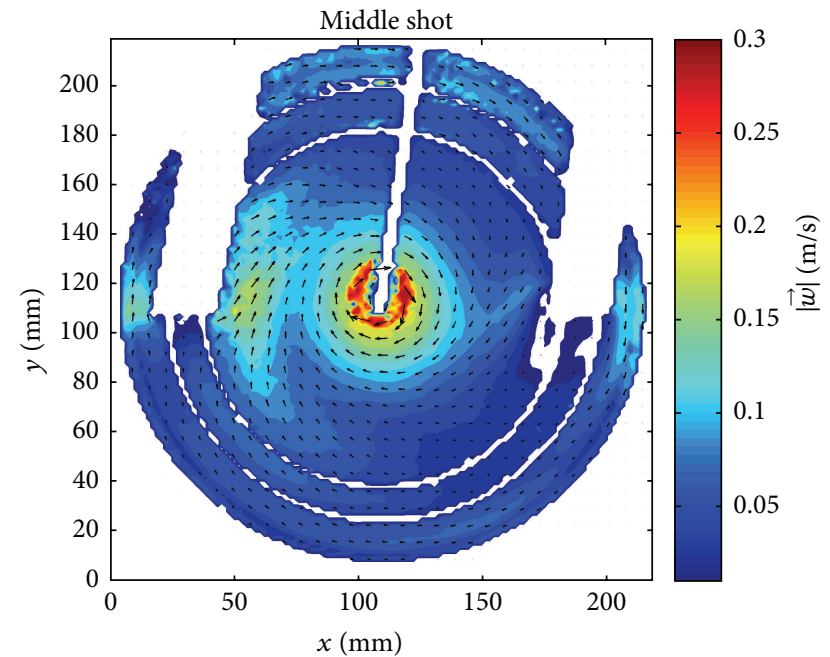

Figure 5: Full velocity field of the horizontal plane at $h / h_{0}=0.45$ (middle shot) of the Rushton turbine stirrer at $400 \mathrm{rpm}$. The light sheet is entering from below in $y$-direction.

characteristics of the flow fields can be found in both parts and are considered to be equal for evaluation.

3.2. Combined Velocity Plots. The intensification of heat transfer as stirring operation intends to reduce the transport limiting boundary layer at the heat exchange surface, as well as to provide the motion of the liquid to and from the coils in terms of convective transport. Besides other influences which affect the boundary layer, for example, the turbulence level, the bulk flow in the vessel is a major criterion to evaluate the performance of a stirring process. Therefore a high level of prospective heat transfer can be concluded from presence and absence of flow regions with high mean velocity. For comparing different stirrer speeds, for each experimental run the dimensionless Reynolds number was calculated:

$$
\operatorname{Re}=\frac{n d_{0}^{2}}{v} .
$$

As a function of stirrer speed $n$, stirrer-blade diameter $d_{0}$, and the kinematic viscosity of the fluid phase $v$, the Reynolds number describes the flow conditions as a relation of inertial and viscous forces. At low Reynolds numbers $\left(\operatorname{Re}<10^{2}\right)$ viscous forces dominate and the flow is described as laminar, whereas at $\mathrm{Re}>10^{2}$ the flow turns into turbulence. In mixing processes high Reynolds numbers are generally more desirable but also accompanied by a high energy demand of the stirrer.

To gain a comprehensive overview of the flow conditions in the reactor, the flow fields of the three different shots are arranged vertically with respect to their bottom clearance to a combined plot. With the described axis symmetry along the baffles, only the unshadowed flow fields of the front part are shown. In the combined plot, each of the three single velocity plots was received out of a separate experimental run at the designated position but recorded with the same settings.

Furthermore the applied technique of 2D-PIV allows the measurement of only two velocity components. Here, for each viewpoint in $x$-and $y$-directions, the coils cover a major part of the vessel, making it impossible to take an unhindered image of the vessel. The third out-of-plane component (in this case the vertical $z$-component) is not readily available, which will be considered when interpreting the $2 \mathrm{D}$-velocity data.

3.3. Rushton Turbine. The Rushton turbine, pictured in Figure 4(a), is a high speed radial-flow stirrer. Due to its high shear levels, this stirrer is primarily used for blending, improving heat transfer, and gas dispersion processes. In the generic radial-flow pattern in a stirred vessel without inserts, the fluid is accelerated in radial direction and deflected at the 
vessel wall into an upper and lower recirculation loop. This stirrer is most effective in low-viscosity liquids and baffled vessels. In case of higher viscosities the fluid is set in primarily tangential motion, according to Zlokarnik [22].

Figure 6 shows the combined velocity plot of the Rushton turbine at $500 \mathrm{rpm}$ and $\mathrm{Re}=2.4 \cdot 10^{4}$. The low shot at $h / h_{0}=0.17$ is closely located below the stirrer at $h_{1} / h_{0}=0.26$ and the lower end of the coils at $h_{C} / h_{0}=0.2$. It shows a clockwise directed circulation pattern governing most of the cross-section. Only flow areas before and behind baffles show flow distortions and a low average velocity. The central region below the area swept by the moving impeller shows an intense tangential circulation flow. Outside the central region the tangential velocity component is decreasing and passing into a stronger radial velocity component.

Middle and top shots in Figure 6 at $h / h_{0}=0.45$ and $h / h_{0}=0.82$, respectively, intersect with the helical pipes, recognizable on the inner rings of missing velocity vectors. The overall intensities are relatively low, compared to the low shot. Tangentially directed velocity vectors are dominating. Especially the outer regions between coils and vessel walls show very low tangentially directed flow velocities without any radial penetration of the coils. Zlokarnik [22] pointed out that the circulation of radial stirrers is strongly deflected by helical coils with an insufficient annulus between coil and wall. Here, the alternating arrangement of helical coils acts as a significant hindrance, leading to a shortened recirculation loop and leaving the outer regions untouched. At the baffles, the tangential flow is split up horizontally into two streams, causing a long trail of stagnant fluid in the gap between both coils. In the middle shot, the upper recirculation loop causes an increasing radial-flow which is directed inwards to the center. At the stirrer shaft, the recirculation ends in an intense whirl along the stirrer shaft back to the stirrer blade.

The impact of stirrer speed is illustrated in Figure 7, showing middle shot plots from 300 to $600 \mathrm{rpm}$. Deviating from earlier plots, the color bar is adjusted to a maximum velocity magnitude of $0.2 \mathrm{~m} / \mathrm{s}$ in order to cover the larger velocity dynamics of different stirrer speeds. As expected, the general flow pattern does not change, but regions of considerably increasing velocity help identify major flows. Based on this it can be concluded whether an adjustment of stirring speed is feasible to affect the heat transfer in the target regions or not.

At the lowest stirrer speed of $300 \mathrm{rpm}$ and $\mathrm{Re}=1.5 \cdot 10^{4}$, turbulence flow conditions are already completely developed for a baffled vessel. From 300 to $400 \mathrm{rpm}$ only minimal changes occur, when the intensity of the redirected flow behind the baffles increases. Important changes take place at the annulus of outer coil and vessel wall, where the flow increases significantly, whereas the inner flow structures change less in intensity. This resulting stronger tangential flow in the outer annulus is marked by a sharp velocity drop towards the middle annulus of both coils. The stagnant region behind the baffles remains unaffected by higher stirrer speed and extends until the quarter circumference. After this distance the tangential flow expands to the middle annulus. In case of fully effective baffling-a common vessel design criteria achieved by four vertically arranged baffles
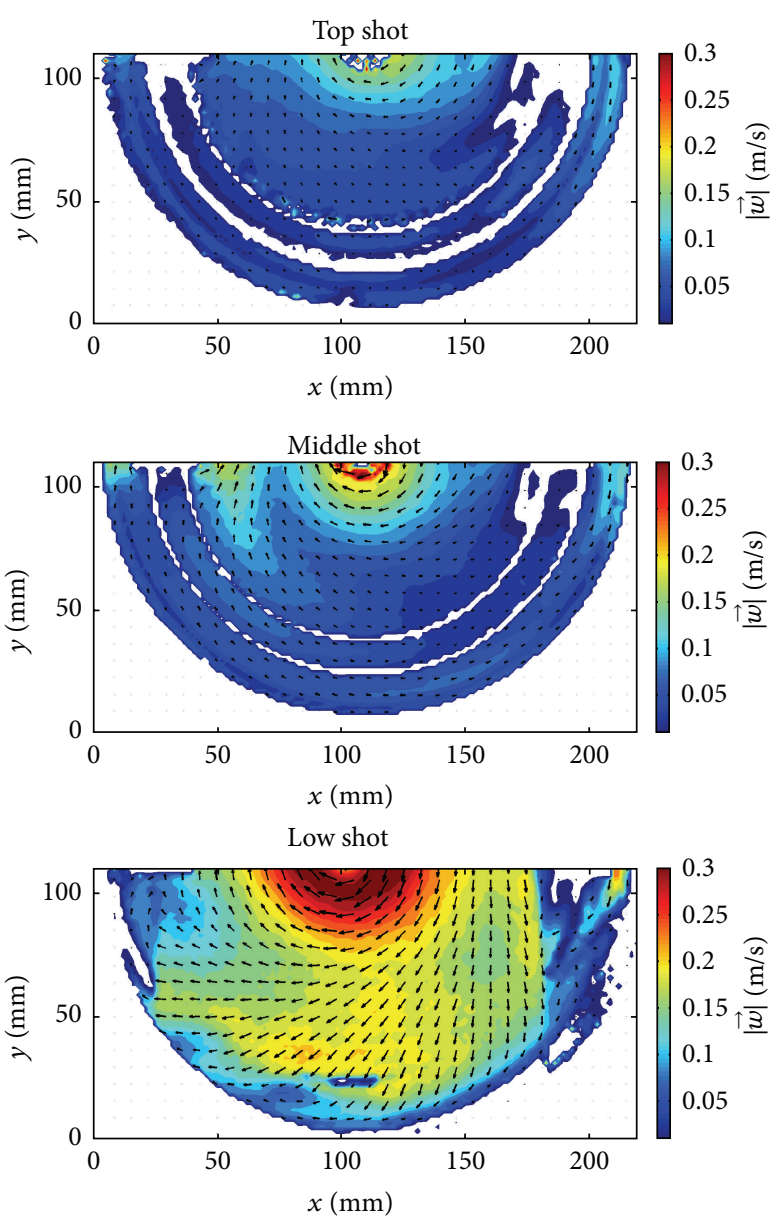

FIGURE 6: Combined velocity plot for the Rushton turbine at $500 \mathrm{rpm}$.

to suppress bulk rotation of the liquid — this stagnant region would incorporate the full middle annulus. Besides baffles are necessary as mount for helical coils, they inhibit a full utilization of heat exchange area significantly. This poor occupation of the middle annulus is problematic, because large amounts of heat exchange area provided by the coils will be wasted.

The last subplot at $600 \mathrm{rpm}$ in Figure 7 points out that primary flow occupation of the coils is given by a tangential flow without radial component. A cross-flow through the coils from the radial-flow Rushton turbine is not observed. In fact, due to the circular coils, the major flow is guided in a tangential pattern, which is typically observed when stirring in high viscous liquids.

3.4. Propeller Stirrer. The combined velocity plots of the propeller stirrer at $500 \mathrm{rpm}$ and $\mathrm{Re}=2.6 \cdot 10^{4}$ are shown in Figure 8 . This stirrer, pictured in Figure 5(b), is usually also operated at high frequencies in low-viscosity liquids and generates an axial-flow pattern. By down-pumping the liquid in the vessel center, a large circulation loop is generated with an 

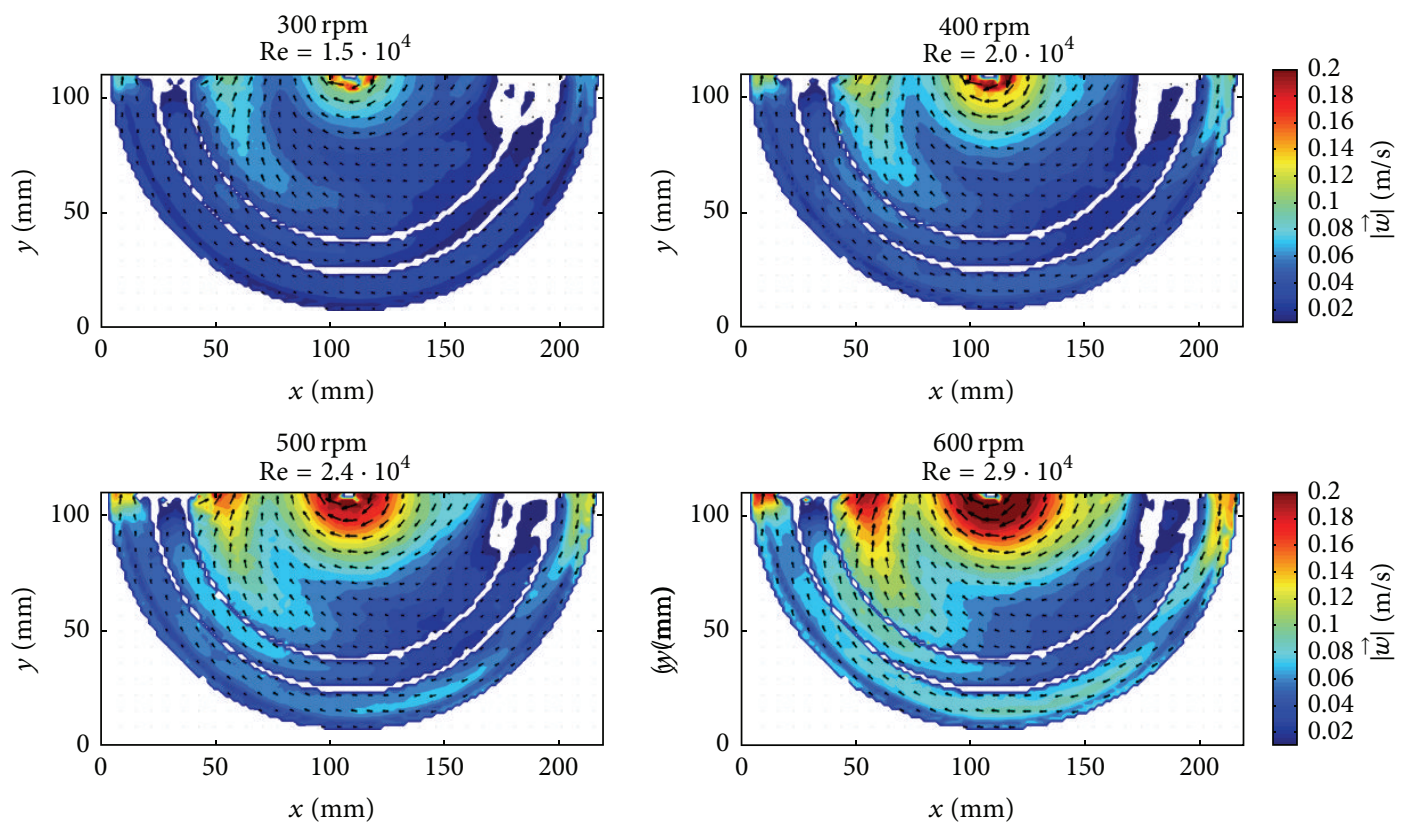

Figure 7: Middle shot $\left(h / h_{0}=0.45\right)$ velocity plots of the Rushton turbine stirrer from 300 to $600 \mathrm{rpm}$.
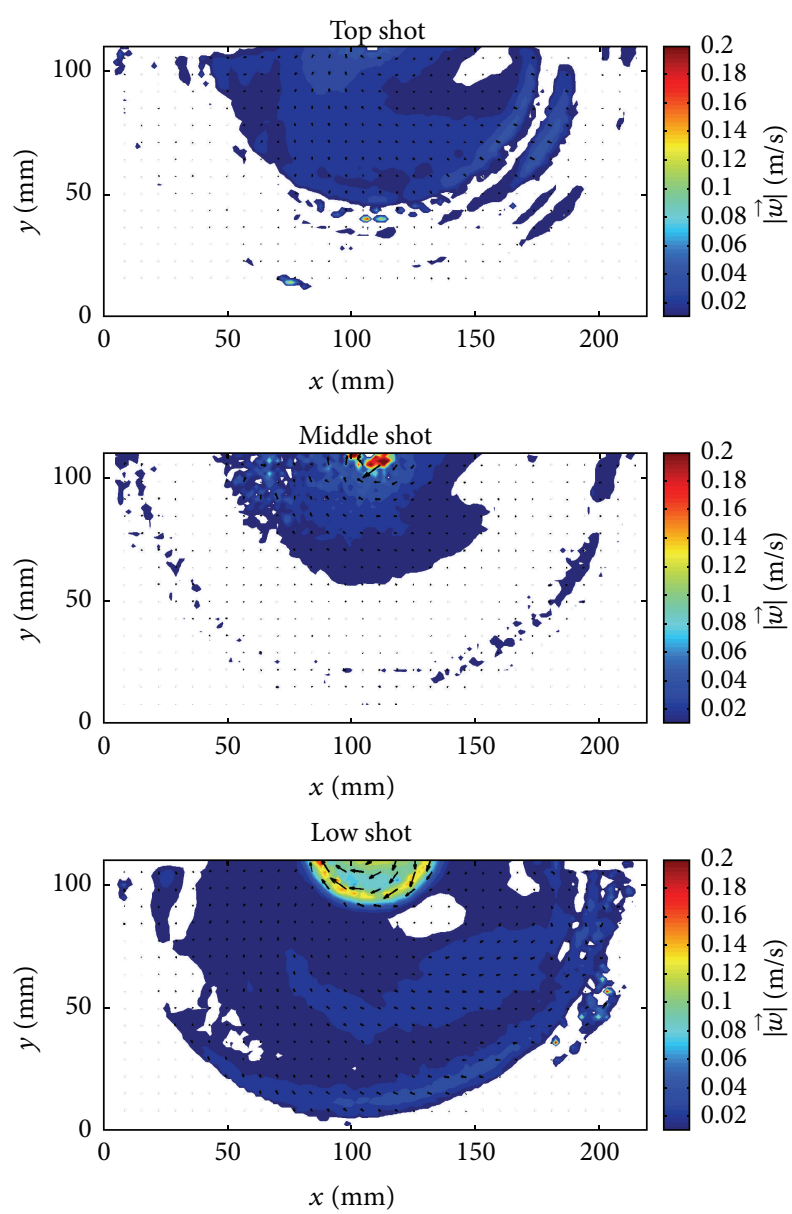

FIgURE 8: Combined velocity plot for the propeller stirrer at $500 \mathrm{rpm}$. upstream at the vessel walls. The field of application is homogenization and suspension of solids.

The low shot is relatively poor in velocity intensity, but for most of the observed area velocity vectors were found. In the central region, a considerable circular flow is recognizable, delimited by a sharp velocity drop outside the area swept by the stirrer.

The middle shot is characterized by a noticeable absence of velocity vectors in the horizontal plane. Only the inner regions show minimal fluid motions, caused by the near stirring blade. At this height, the flow pattern within the reactor consists of a downstream in the center and an upstream in the outer regions, separated by the gap between coils and vessel wall. Most flow activities take place in the vertical plane and will be found in the invisible vertical or out-of-plane component. Accordingly, the top shot is also very poor in velocity intensity but minimally higher than middle shot. Broader areas of low horizontal velocity flows indicate the upper part of the recirculation loop.

For comparing different stirrer velocities, the more descriptive low shot of the propeller stirrer is chosen in Figure 9. With a minimal Re of $1.6 \cdot 10^{4}$ at $300 \mathrm{rpm}$ the formal criteria of turbulence flow are also fulfilled. Nevertheless outside the narrow field of the stirrer, the velocity magnitude is marginal with about $0.02 \mathrm{~m} / \mathrm{s}$. For large parts of the flow field, no valid velocity vectors were found. At the highest stirrer velocity with $\mathrm{Re}=3.1 \cdot 10^{4}$ most velocity vectors are valid, but the overall velocity in the horizontal plane remains poor. Only the central swirl below the stirrer blade gains intensity. With knowledge of a noteworthy absence of fluid movement in the horizontal plane and a theoretically high Reynolds number, it can be concluded that most flow activities take place in axial direction. 

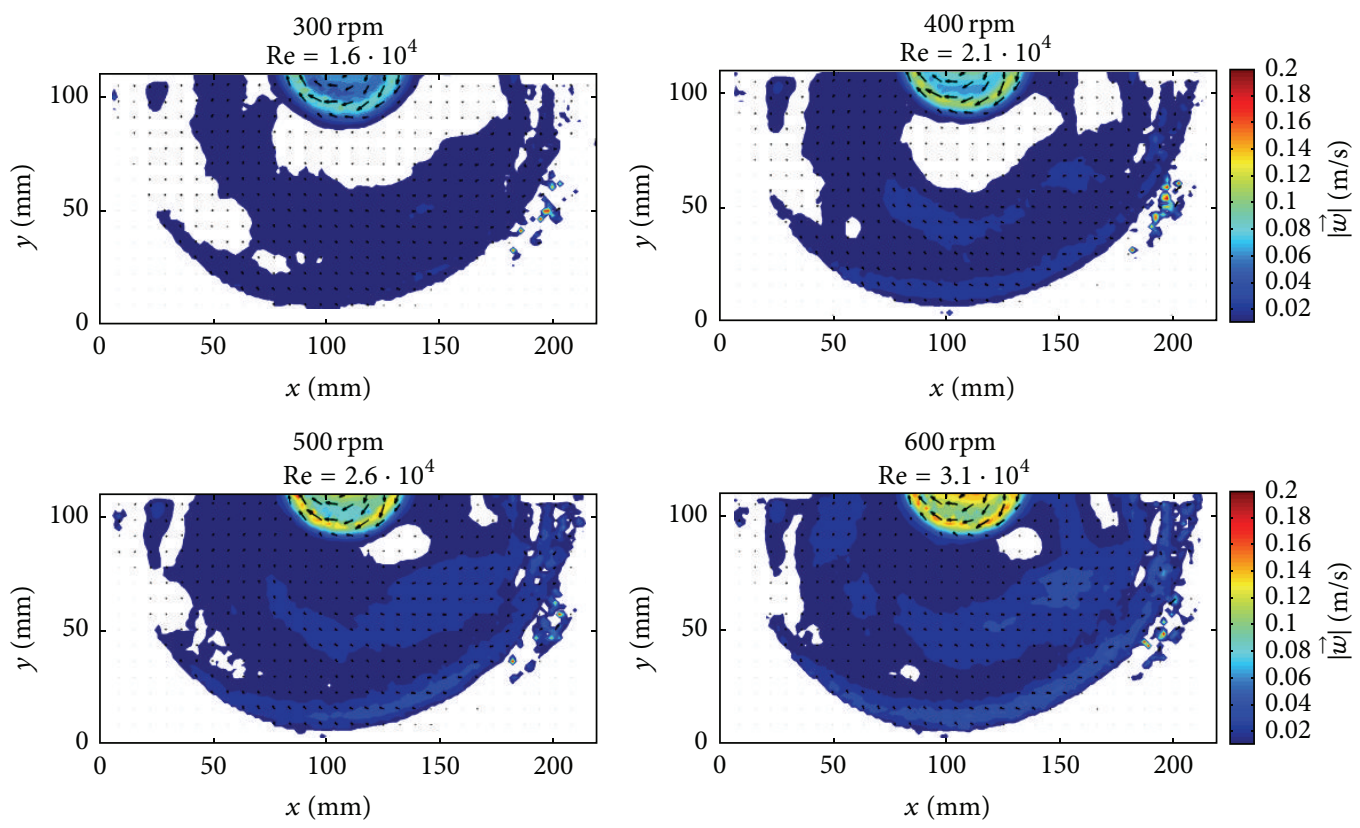

FIGURE 9: Low shot $\left(h / h_{0}=0.2\right)$ velocity plots of the propeller stirrer from 300 to $600 \mathrm{rpm}$.

\section{Summary and Conclusions}

2D-PIV technique was applied to investigate the flow conditions in the horizontal plane in stirred vessels with inserts of two helical coils. Two different stirrers (radial-flow Rushton turbine and axial-flow propeller) were tested in the range from 300 to $600 \mathrm{rpm}$ for their flow-pattern to provide sufficient heat transfer from helical coils. The main advantage of the experimental setup in this work was that despite the considerable optical hindrance of two alternating helical coils the complete velocity field of the horizontal cross-section was captured. Single plots of three different positions in the vessel were arranged in combined velocity plots to allow a comprehensive overview.

Inserts (such as helical coils) usually induce disturbances and deflect main flows in other directions. It was found that the created flow pattern of the actual radial-flow Rushton turbine is dominated by tangential flow structures, as known from high-viscosity liquids. Cross-flow structures through coils cannot be observed; contact with heat exchange area is maintained by flow in longitudinal direction of the pipes. Behind baffles a long trail of stagnant fluid is created, leaving large amounts of heat transfer area of the inner annulus untouched.

For the axial-flow propeller stirrer, only the deflection at the vessel bottom from a central downstream to an upstream at the vessel walls was adequately observed. Because most flow characteristics of an axial-flow pattern cannot be captured in the horizontal plane, the found velocities are generally very low. Even though the experimental design does not fit the characteristics of an axial-flow pattern, general information about the flow structures around coils vessels can be concluded. For this case of an axial-flow stirrer, more experimental designs are necessary to make statements of fluid movement in the vertical plane. A combined representation of horizontal and vertical velocity information is in preparation. Therefore the combination of the given results with CFD simulations seems to be a very promising approach to (1) complete the fragmented data due to the hindered optical access, (2) add information on the third out-of-plane component, and (3) validate the results by an independent measurement technique. The first results of this work indicate that the current dimension criteria for the right stirrer type with helical heat exchanger coils should be reconsidered. Further investigations should lead to deeper constructive and flowreferring advices to improve the heat exchange around helical coils in stirred tanks.

\section{Conflict of Interests}

The authors declare that there is no conflict of interests regarding the publication of this paper.

\section{References}

[1] G. B. Tatterson, R. S. Brodkey, and R. V. Calabrese, "Move mixing technology into the 21st century," Chemical Engineering Progress, vol. 87, no. 6, pp. 45-48, 1991.

[2] T. H. Chilton, T. B. Drew, and R. H. Jebens, "Heat transfer coefficients in agitated vessels," Industrial \& Engineering Chemistry, vol. 36, no. 6, pp. 510-516, 1944.

[3] S. N. M. Nagata and T. Takhimoto, "Turbulent heat transfer from helical cooling coils in agitated vessels," Heat Transfer, vol. 1, pp. 49-56, 1972.

[4] W. T. Appleton and W. C. Brennan, "Some observations on heat transfer to agitated liquids," The Canadian Journal of Chemical Engineering, vol. 44, no. 5, pp. 276-280, 1966. 
[5] J. Y. Oldshue, "Mixing theory and practice, V. M. Uhl and J. B. Gray Vol. I, Academic Press, New York (1966). 340 pages, \$15.50," AIChE Journal, vol. 13, no. 4, pp. 826-827, 1967.

[6] A. H. P. Skelland and G. R. Dimmick, "Heat transfer between coils and non-newtonian fluids with propeller agitation," Industrial and Engineering Chemistry Design and Development, vol. 8, no. 2, pp. 267-274, 1969.

[7] R. Poggemann, A. Steiff, and P.-M. Weinspach, "Wärmeübergang in Rührkesseln mit einphasigen Flüssigkeiten," Chemie Ingenieur Technik, vol. 51, no. 10, pp. 948-959, 1979.

[8] M. Kraume, Mischen und Rühren: Grundlagen und Moderne Verfahren, Wiley-VCH, Weinheim, Germany, 2003.

[9] V. T. Perarasu, M. Arivazhagan, and P. Sivashanmugam, "Heat transfer studies in coiled agitated vessel with varying heat input," International Journal of Food Engineering, vol. 7, no. 4, article 3, 2011.

[10] R. J. Adrian, "Twenty years of particle image velocimetry," Experiments in Fluids, vol. 39, no. 2, pp. 159-169, 2005.

[11] J. Sheng, H. Meng, and R. O. Fox, "Validation of CFD simulations of a stirred tank using particle image velocimetry data," Canadian Journal of Chemical Engineering, vol. 76, no. 3, pp. 611625, 1998.

[12] K. J. Myers, R. W. Ward, and A. Bakker, "A digital particle image velocimetry investigation of flow field instabilities of axial-flow impellers," Journal of Fluids Engineering, vol. 119, no. 3, pp. 623632, 1997.

[13] A. Bakker, K. J. Myers, R. W. Ward, and C. K. Lee, "The laminar and turbulent flow pattern of a pitched blade turbine," Transactions of IChemE, vol. 74, pp. 485-491, 1996.

[14] D. F. Hill, K. V. Sharp, and R. J. Adrian, "Stereoscopic particle image velocimetry measurements of the flow around a Rushton turbine," Experiments in Fluids, vol. 29, no. 5, pp. 478-485, 2000.

[15] K. V. Sharp and R. J. Adrian, "PIV study of small-scale flow structure around a Rushton turbine," AIChE Journal, vol. 47, no. 4, pp. 766-778, 2001.

[16] N. G. Deen and B. H. Hjertager, "Particle image velocimetry measurements in an aerated stirred tank," Chemical Engineering Communications, vol. 189, no. 9, pp. 1208-1221, 2002.

[17] D. Hill, D. Troolin, G. Walters, W. Lai, and K. V. Sharp, "Volumetric 3-component velocimetry (V3V) measurements of the turbulent flow in stirred tank reactors," in Proceedings of the 14th International Symposium on Applications of Laser Techniques to Fluid Mechanics, Lissabon, Portugal, 2008.

[18] Y. Wang, Q. Rao, J. Fan, and W. Fei, "PIV measuremetns and CFD simulation of viscous fluid flow in a stirred tank agitated by a Rushton turbine," in Proceedings of the 5th International Conference on CFD in the Process Industries, Melbourne, Australia, 2006.

[19] R. Atibeni, Z. Gao, and Y. Bao, "Effect of baffles on fluid flow field in stirred tank with floating particles by using PIV," The Canadian Journal of Chemical Engineering, vol. 91, pp. 570-578, 2012.

[20] P. S. Harvey and M. Greaves, "Turbulent flow in an agitated vessel: part I: predictive model \& part II: numerical solution and model prediction," Transactions of IChemE, vol. 60, pp. 195-210, 1982.

[21] N. C. Markatos, "Computer simulation of turbulent fluid flow in chemical reactors," Advances in Engineering Software, vol. 5, no. 1, pp. 32-38, 1983.

[22] M. Zlokarnik, Rührtechnik: Theorie und Praxis, Springer, Berlin, Germany, 1999. 

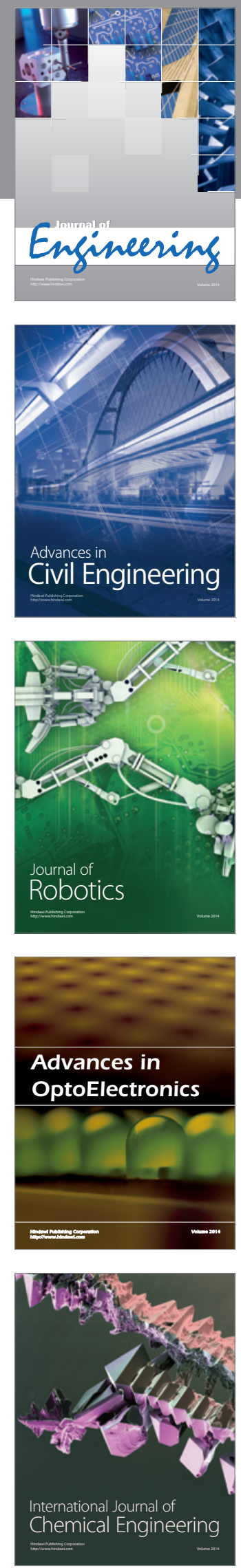

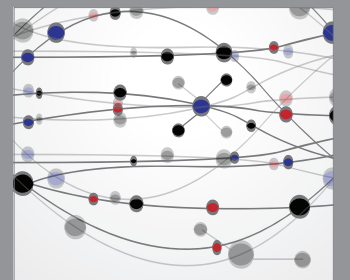

The Scientific World Journal
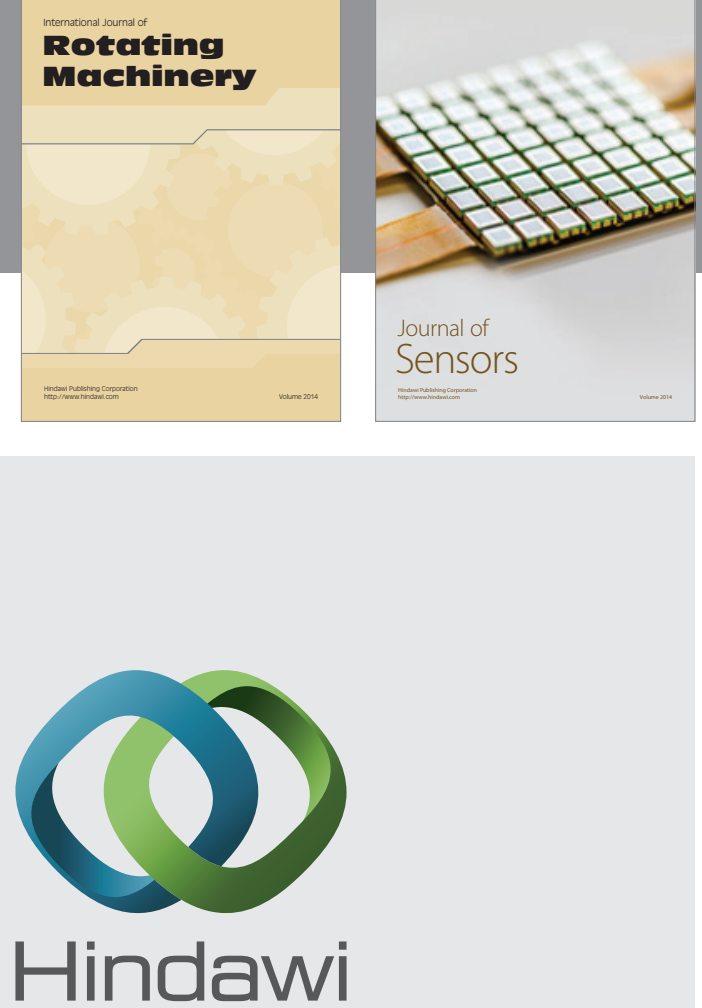

Submit your manuscripts at http://www.hindawi.com
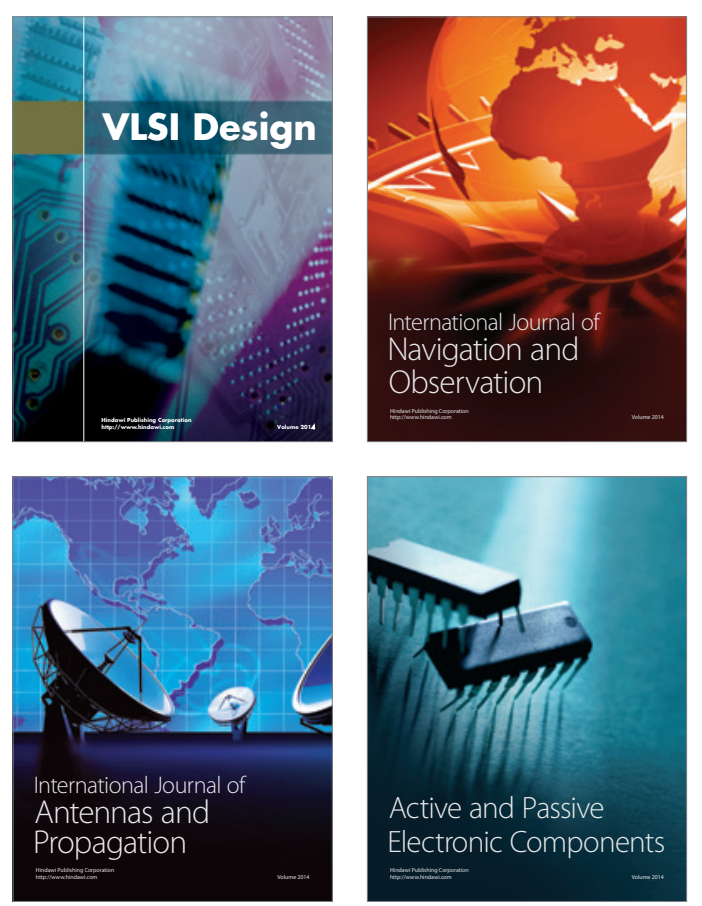
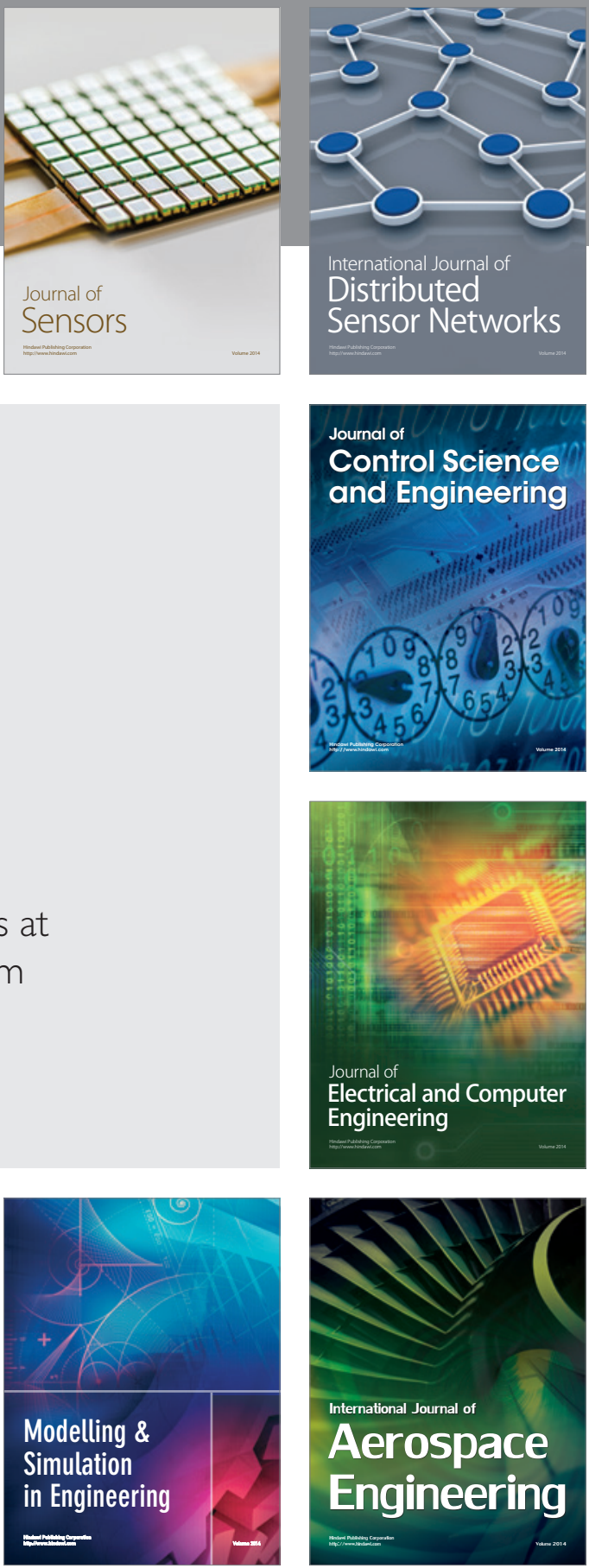

Journal of

Control Science

and Engineering
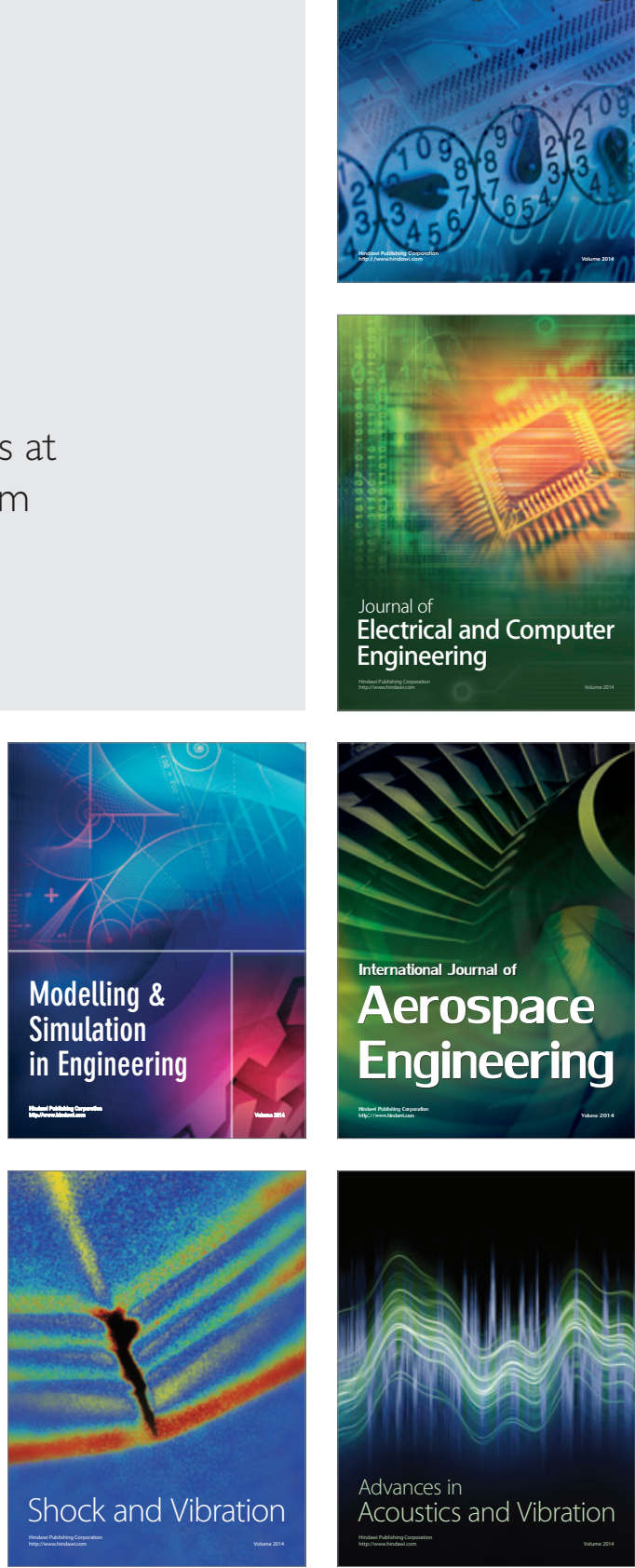